Software solutions manage the definition, operation, maintenance and configuration control of the National Ignition Facility

D. Dobson, A. Churby, E. Krieger, D. Maloy, K. White

July 29, 2011

IAEA 8th Technical Meeting

San Francisco, CA, United States

June 20, 2011 through June 24, 2011 
This document was prepared as an account of work sponsored by an agency of the United States government. Neither the United States government nor Lawrence Livermore National Security, LLC, nor any of their employees makes any warranty, expressed or implied, or assumes any legal liability or responsibility for the accuracy, completeness, or usefulness of any information, apparatus, product, or process disclosed, or represents that its use would not infringe privately owned rights. Reference herein to any specific commercial product, process, or service by trade name, trademark, manufacturer, or otherwise does not necessarily constitute or imply its endorsement, recommendation, or favoring by the United States government or Lawrence Livermore National Security, LLC. The views and opinions of authors expressed herein do not necessarily state or reflect those of the United States government or Lawrence Livermore National Security, LLC, and shall not be used for advertising or product endorsement purposes. 


\title{
Software solutions manage the definition, operation, maintenance and configuration control of the National Ignition Facility
}

\author{
Darwin Dobson $^{\mathrm{a}}$, Al Churby ${ }^{\mathrm{a}}$, Ed Krieger $^{\mathrm{a}}$, Donna Maloy ${ }^{\mathrm{a}}, \mathrm{Kevin}_{\text {White }}{ }^{\mathrm{a}}$ \\ ${ }^{a}$ Lawrence Livermore National Laboratory, Livermore, CA, USA
}

\begin{abstract}
The National Ignition Facility (NIF) is the world's largest laser composed of millions of individual parts brought together to form one massive assembly. Maintaining control of the physical definition, status and configuration of this structure is a monumental undertaking yet critical to the validity of the shot experiment data and the safe operation of the facility. The NIF business application suite of software provides the means to effectively manage the definition, build, operation, maintenance and configuration control of all components of the National Ignition Facility. State of the art Computer Aided Design software applications are used to generate a virtual model and assemblies. Engineering bills of material are controlled through the Enterprise Configuration Management System. This data structure is passed to the Enterprise Resource Planning system to create a manufacturing bill of material. Specific parts are serialized then tracked along their entire lifecycle providing visibility to the location and status of optical, target and diagnostic components that are key to assessing pre-shot machine readiness. Nearly forty thousand items requiring preventive, reactive and calibration maintenance are tracked through the System Maintenance \& Reliability Tracking application to ensure proper operation. Radiological tracking applications ensure proper stewardship of radiological and hazardous materials and help provide a safe working environment for NIF personnel.
\end{abstract}

Keywords: Computer Aided Design, Configuration Management, Change Control, Data Replication, Enterprise Resource Planning, Enterprise Asset Management, Radiological Inventory Management

\section{Introduction}

The National Ignition Facility (NIF) at the Lawrence Livermore National Laboratory (LLNL) is a stadiumsized structure that contains a 192-beam, 1.8-Megajoule, 500-Terawatt, ultraviolet laser system together with a 10-meter diameter target chamber with room for multiple experimental diagnostics. NIF is the world's largest and most energetic laser experimental system, providing a scientific center to study inertial confinement fusion (ICF) and matter at extreme energy densities and pressures. NIF's laser beams are designed to compress fusion targets to conditions required for thermonuclear burn, liberating more energy than required to initiate the fusion reactions. Maintaining control of the physical definition, status and configuration of millions of individual and assembled parts is a monumental undertaking yet critical to the validity of the shot experiment data and the safe operation of the facility.

The advancement of complex software solutions play a significant role in simplifying the task of tracking and controlling the physical configuration of any given product or system. NIF has used an integrated approach to deploy a suite of software, primarily commercial software products that provide the means to effectively and safely manage the definition, build, operation, maintenance and configuration control of all components and materials that make up the facility. Software that is designed to help manage definition and change control should support the key tenets of configuration and change control:
- Capable of capturing and maintaining an accurate physical description of the system

- Promote a single source of data to reduce the need for replication

- Ability to track changes and their impact on surrounding systems

- Visibility into what components are installed in a given location at a given time

- Promote a safe environment

While software plays a significant role in supporting the task of change and configuration control, it is important to note that software can only supplement a well defined and followed configuration management and change control policy.

\section{Physical Definition of NIF}

NIF utilizes a suite of commercial Computer Aided Design (CAD), Analysis, and Data Management software applications for the three dimensional virtual definition of the facility. The implementation and utilization of this suite of applications has aided in making the physical NIF a reality. As NIF is now an operational facility, software applications like these and others will be key in collaborating with other institutions seeking to run experimental setup at the NIF facility.

Among the suite of engineering tools are mathematical applications used to facilitate engineering solutions for the scientific design challenges of NIF. The output of these mathematical solutions provides important design criteria for the product being 
developed. The process of capturing a virtual design begins by creating a model for each individual component within the facility. These individual models are joined to create virtual assemblies of all of the NIF subsystems. NIF's designers and engineers ensure the fit and function of the millions of objects that make up the facility by using the virtual models to run interference checks. In addition, finite element analysis applications are used to ensure that the components and subassemblies that comprise NIF are designed to withstand the mechanical and thermal stresses introduced by the operation of the system. A CAD data management application that works in conjunction with NIF's modeling packages enables concurrent design and a single point data storage location for all of the designers and engineers. This application manages over 40 million relationships that exist between all of the 3D models and also tracks design iterations and history.

Once modeled, the design and its components and sub-assemblies are documented through the use of engineering drawings. Engineering drawings provide a visual capture of the design and a list or bill of material of the components that make up the design. The relational nature of the software maintains a one to one capture of the modeled assembly and the bill of material found on the drawing. The CAD model and its associated information becomes the data source for many downstream applications and consumption. (See Fig. 1) The integrity and accuracy of this data is of critical importance.

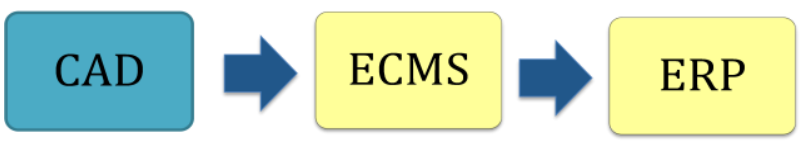

Fig. 1

The CAD data management application is integrated with LLNL's institutional Configuration Management system. Together they control the initial creation, approval, release, and revision of every $3 \mathrm{D}$ model and drawing associated with NIF.

\section{Data Structure and Formal Change Control}

Once the virtual design is finalized and ready for review, approval and release, the individual part information and the assembly data structure is passed electronically from the CAD data management system into the Enterprise Configuration Management System (ECMS). This electronic data transfer removes human intervention and significantly reduces errors thus maintaining the accuracy of the data.

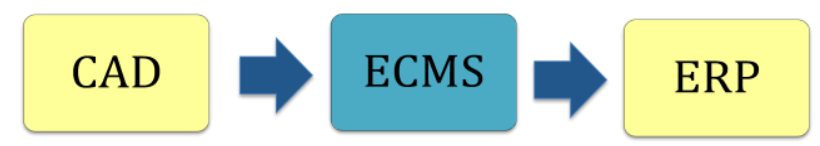

Fig. 2
Here, product design information is made available to other NIF groups and functions outside of engineering such as procurement, assembly technicians, production control, NIF operations, etc. for final review and approval. ECMS is also where the 'as designed' configuration is captured through the use of the officially released Engineering Bill of Material (EBOM). Once the design is approved and signatures acquired, it is released for production use. A released design cannot undergo any changes without prior approval.

Change control is a primary function of ECMS and the system has been configured to allow for different levels of change control rigor depending of the level of impact as shown in Fig. 3.

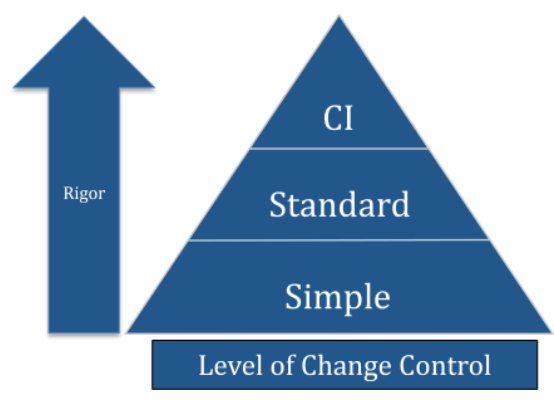

Fig. 3

A Simple change typically has no functional or systematic impact and therefore requires no formal review prior to creating a new revision and implementing the change. A Standard change does have impact on the form, fit or function of the system and or surrounding systems. This requires the initiation of a formal change request where the proposed change is documented and reviewed for functional impact. Once change approval is obtained, the modification to the design can be implemented. A Configuration Item (CI) change proposal requires an additional level of analysis and review. Configuration Items are systems whose failure could have negative impact on environmental and worker safety, and thus, require the higher level of scrutiny when changes are proposed. ECMS has been configured to provide visual indicators to alert users when dealing with CI's. In addition, the need for increased rigor in change analysis and review is systematically imposed such that individuals responsible for the configuration and control of the CI must be included in the change approval process.

When change approval is obtained and implemented, the updated design is released in ECMS. Upon release, part and EBOM information is systematically transferred into the NIF Enterprise Resource Planning system.

\section{Tracking the Assembly and Installation}

The NIF Enterprise Resource Planning (ERP) system is a software application used to manage the assembly, installation, and maintenance of specialized laser and diagnostic equipment. NIF ERP implements the familiar concepts of enterprise resource management using 
product definition and time phased management of demand and supply. Demand and supply requirements are managed over time. Labor, equipment, and material resources are defined and managed.

The product lifecycle in NIF ERP begins with product planning. From the NIF project management tools, high level product definitions and installation dates are established. These are used to create demand in the form of planned orders in ERP. The next phase in the product lifecycle is product definition, which begins with ECMS creating parts and assemblies in ERP via the production release process. Upon release from ECMS, information is transferred electronically to the ERP system. (Fig. 4)

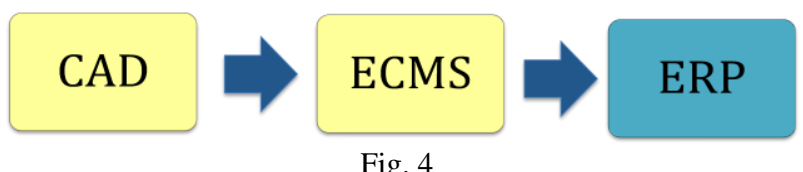

The ERP master catalog is built from the bottom up by first creating child parts, then adding parent parts, and finally bringing these parts together into assemblies. As assemblies are released through engineering systems, EBOM records are transferred from the CAD source data through ECMS to ERP. The fully automated nature of the part and BOM transfer process ensures that the ERP master catalog and EBOM data are efficiently maintained, and are both accurate and current.

Production Control Engineers begin their part of the product lifecycle with the released EBOM and add intelligence required for the assembly and installation of end products. MBOMs are created by adding production routings to EBOMs. The routings contain the labor and machine resource information required for assembly. Specific parts in MBOMs are flagged for tracking by serial number based on safety significance, operational significance, and calibration and maintenance requirements. Product codes and other attributes are assigned to facilitate queries, reports, and use of ERP data by other management systems.

Execution of product assembly and installation using NIF ERP is similar to commercial production processes. A Master Production Schedule (MPS) is run on a daily basis to create and schedule assembly and subassembly work orders. Material Requirements Planning (MRP) is run daily to assess supply status and manage the material acquisition process. Capacity planning is used in conjunction with MPS to ensure proper equipment utilization without overloading labor and machine resources. Shortage and exception reporting are reviewed daily to manage supply chain issues. As work orders are released and material, labor, and machine resources are applied to complete them, detailed as-built data is accumulated and archived for each work order processed. Daily reporting on the work order data enables project managers to monitor progress against project plans.
Following completion of product assembly, NIF ERP tracks the finished items through installation at specific facility locations defined by NIF project management tools. Following installation, ERP work orders are used to manage the final testing of each assembly, subsystem, and system. The ERP work orders provide a record of the testing and operational certification of each item of hardware installed in the facility. Work orders for installation and test activities are scheduled, released, completed, and archived. Reporting of installation and test progress against plans is enabled using work order data. Rework, reinstallation and retest of any item of equipment follows a process similar to that of initial production, so that there are complete records of all work done on the equipment installed.

In addition to supporting delivery of installed and operating hardware, NIF ERP is a resource for detailed records of as-built, and as-maintained hardware configuration over time. This hardware configuration data provides visibility into the location and status of key optical, target, and diagnostic components over the lifetime of the operating facility. This information is used to assess system readiness by enabling comparison of the equipment installed and available versus the equipment required for a given experiment.

The hardware configuration data also enables historical analysis of equipment performance by providing a record of the specific items of equipment used in any given experiment in the facility.

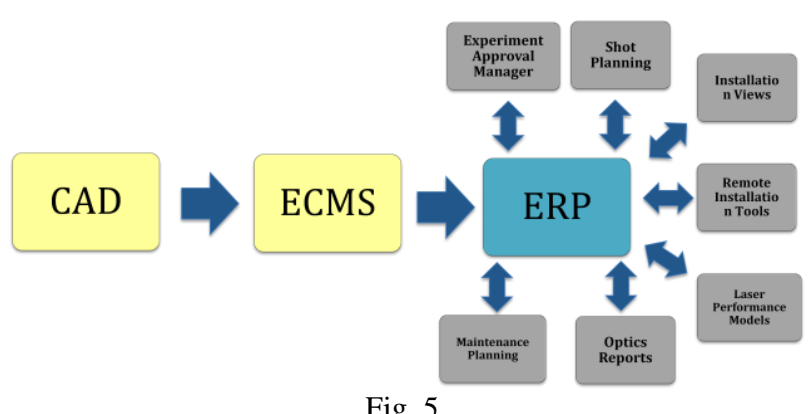

NIF ERP is the primary data hub from which many other applications and reporting tools requiring visibility into the location and status of key components access that information.

\section{Other Key Applications}

\subsection{Managing the Maintenance of NIF}

The NIF System Maintenance and Reliability Tracking (SMaRT) System supports the predictive/preventive, corrective, reactive, and calibration maintenance for NIF operations and facilities management. SMaRT is an implementation of a commercially available Enterprise Asset Management (EAM) application. SMaRT manages the work flow and efforts for over thirty thousand equipment items requiring maintenance across NIF's two hundred plus 
subsystems and thirty eight facilities. This includes nine hundred plus preventive and calibration schedules managing over eight thousand PM and seven thousand RM work orders. SMaRT has integrations to other LLNL institutional and NIF services that include LLNL's training and qualification system for validation and verification of qualifications for employees, NIF ERP for the 180,000 material inventory items needed on work orders, NIF's work permitting and problem logging applications to verify the authorize permitting of work and the tracking of reported problems, ECMS for the document lifecycle management of procedures and tasks, LLNL's people database to verify and deactivate employees/supervisors/users within the application, and other institutional applications to collaborate and validate work flow and information. The SMaRT application ensures the Configured Items (CI's) and safety systems related equipment are under configuration control to ensure work is performed on time and with the latest released procedures to keep the National Ignition Facility running in safe operations and ready for shot experiments. Ensuring the reliability of NIF, including its support systems and utilities, is essential to ensuring the availability of the NIF in its support of laser operations

Preventive Maintenance (PM) consists of all the systematically planned and scheduled actions performed to prevent equipment failure. The PM program defines the required activities and the frequency with which they should be performed. Selections of required PM actions are based on manufacturers' recommendations, industry and NIF experience, and good engineering practice. PM frequency is based on adequately implementing the entire program, considering such elements as predictive maintenance results, vendor recommendations, and monitoring of performance. The NIF maintenance program's applicability and implementation as it is applied to an individual subsystem is documented in the associated System Level Maintenance Plan (SLMP); each NIF's subsystems, equipment, and assets are further categorized into a structured list or Technical Hierarchy. Multiple hierarchies are used to organize and structure NIF's equipment. However, all hierarchies report to one overriding hierarchy, that is the Unified System Hierarchy (USH).

\subsection{Monitoring Hazardous Material}

The Radiological Inventory Management System (RIMS) is one of a suite of applications used to ensure the safety of NIF workers by continuously monitoring the radioactivity levels and their potential negative impact on workers and the facilities.

RIMS is a web-based application that provides the capability to track additions, deletions and specific levels of radioactive isotope inventory in the facility in accordance with DOE and LLNL standards. RIMS maintains current radioactive inventories within the NIF complex including additions from targets, generated yield and shot products and removals to waste, sewer or stack applying a continuous decay algorithm to assure an accurate current inventory. RIMS also tracks yield and specific hazardous materials within the facility to assure compliance with annual regulatory limits. The primary users of RIMS are the NIF Radiological Control Technicians (RCT) who are responsible for entering inventory additions and subtractions into RIMS. The information includes the isotopes, quantities and location or disposition. Documentation, such as isotopic analysis and signed permits for release to sewer can be attached and be associated with the specific inventory transaction.

The Survey Information Management System (SIMS) is one of a suite of applications used to ensure the safety of workers in the NIF by continuously monitoring the radioactivity levels and their potential negative impact on workers and the facilities.

SIMS is a web-based application that provides the capability to record and report information about radiological or Beryllium surveys conducted in the NIF complex. The tool is used by the Radiological Control Technicians (RCT) to enter data about the monitoring processes that occur in the NIF. SIMS supports Radiological Swipe, Air and Liquid Sampling and provides the functionality to upload results from one of several Liquid Scintillation Counters available. SIMS also supports Direct Read Instrument sampling using Dose Rate and Tritium Air type instruments. In the future, other instruments, such as rate meters, REM meters and swipe counters will be supported.

RCTs sample the air, surfaces and instruments to monitor the contamination levels of alpha, beta, tritium or beryllium present in the facility. RCTs record the location of where the samples were taken, the size of the samples, what type of samples were collected and, if applicable, the instrument that was used to collect the sample. The RCTs may also upload associated survey documents, such as maps, diagrams, etc., to be stored in the database. Some sampling is performed in the field using Dose Rate (Ion Chambers, Teletectors, etc.) or Tritium Air instruments. These results are entered directly into SIMS.

Other sampling involves transferring physical samples to the NIF laboratory to analyze. Analysis results data such as the method, instrument used, processing time, efficiencies and raw results are output from the analysis instrument. Final results are calculated from the raw results and uploaded along with the analysis data into SIMS.

Surveys are reviewed or approved online by the Health Physicist. Survey reports with the sample and result information are then generated for distribution to the appropriate Health and Safety personnel. 